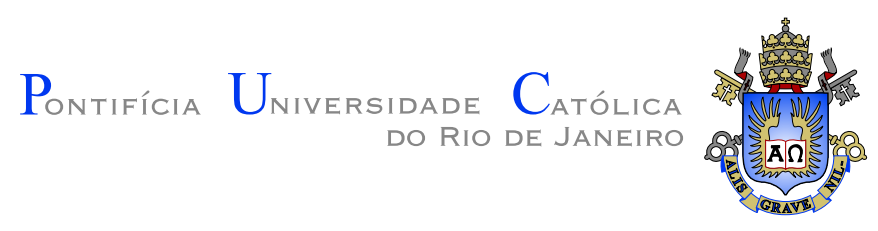

Camila Patrícia Bazílio Nunes

\title{
Avaliação da Modularidade e Estabilidade de Técnicas de Implementação para Linhas de Produtos de Sistemas Multi-agentes
}

\section{Dissertação de Mestrado}

Dissertação apresentada como requisito parcial para obtenção do grau de Mestre pelo Programa de Pós-graduação em Informática do Departamento de Informática da PUC-Rio

Orientador : Prof. Carlos José Pereira de Lucena Co-Orientador: Prof. Uirá Kulesza 


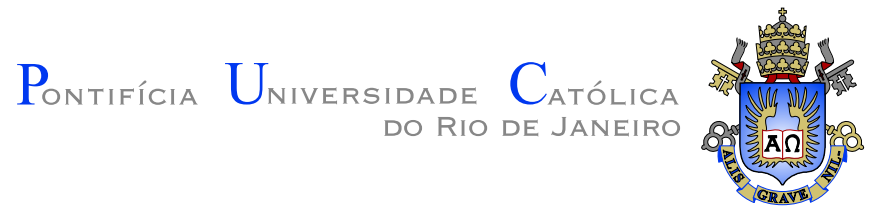

Camila Patrícia Bazílio Nunes

\section{Avaliação da Modularidade e Estabilidade de Técnicas de Implementação para Linhas de Produtos de Sistemas Multi-agentes}

Dissertação apresentada como requisito parcial para obtenção do grau de Mestre pelo Programa de Pós-graduação em Informática do Departamento de Informática do Centro Técnico Científico da PUC-Rio. Aprovada pela Comissão Examinadora abaixo assinada.

Prof. Carlos José Pereira de Lucena Orientador Departamento de Informática - PUC-Rio

Prof. Uirá Kulesza Co-Orientador Departamento de Informática e Matemática Aplicada - UFRN

Prof. Arndt von Staa Departamento de Informática - PUC-Rio

Prof. Renato Fontoura de Gusmão Cerqueira Departamento de Informática - PUC-Rio

Prof. José Eugenio Leal Coordenador Setorial do Centro Técnico Científico - PUC-Rio 
Todos os direitos reservados. É proibida a reprodução total ou parcial do trabalho sem autorização da universidade, do autor e do orientador.

\section{Camila Patrícia Bazílio Nunes}

Graduou-se em Ciência da Computação na Universidade Federal de Alagoas em 2006. Atualmente atua na área de Linha de Produtos, Engenharia de Software Empírica e Desenvolvimento de Software Orientado a Aspectos no Laboratório de Engenharia de Software (LES) da PUC-Rio.

Ficha Catalográfica Nunes, Camila

Avaliação da Modularidade e Estabilidade de Técnicas de Implementação para Linhas de Produtos de Sistemas Multiagentes / Camila Patrícia Bazílio Nunes; orientador: Carlos José Pereira de Lucena; co-orientador: Uirá Kulesza. - Rio de Janeiro: PUC-Rio, Departamento de Informática, 2009.

$118 \mathrm{f}$; $30 \mathrm{~cm}$

1. Dissertação (mestrado) - Pontifícia Universidade Católica do Rio de Janeiro, Rio de Janeiro, 2009.

Inclui referências bibliográficas.

1. Informática - Tese. 2. Linhas de Produto de Software. 3. Sistemas Multi-agentes. 4. Estudo Empírico. 5. Técnicas de Implementação. 6. Projeto de Software. I. Lucena, Carlos. II. Kulesza, Uirá. III. Pontifícia Universidade Católica do Rio de Janeiro. Departamento de Informática. IV. Título. 


\section{Agradecimentos}

Primeiramente, agradeço a Deus por me conceder sempre momentos maravilhosos. Só tenho a agradecer todos esses anos da minha vida, por todas as conquistas.

À minha família querida, meus pais, Julio e Girleide, meus irmãos, Sady e Leonardo. Sem eles, esse trabalho não teria sido concluído. Amo vocês.

Durante os dois anos de mestrado, tive a oportunidade de conhecer pessoas especiais, que me proporcioram grandes oportunidades e me ensinaram bastante.

Professor Carlos Lucena, meu orientador, com quem eu tive a oportunidade de aprender e interagir, tornando o trabalho mais gratificante. Sempre me manteve motivada no trabalho e me concedeu oportunidades únicas. Ao senhor, toda a minha consideração e respeito.

Uirá Kulesza, meu orientador, assim o considero por toda sua dedicação e empenho. Durante este tempo de convivência, posso dizer que ele também se tornou um amigo. Obrigada pelas discussões, pelas dicas, pela amizade e pelas seções de análise, que foram muito importantes durante os momentos de baixo astral :). Uirá foi a pessoa que primeiro me acolheu no LES. Nossas conversas foram muito importantes para o meu crescimento pessoal e profissional. Valeu moral!!!

Ingrid, minha parceira de trabalho desde o início de mestrado. Com ela tive a oportunidade de aprender muito. Fizemos um trabalho em equipe que deu certo, fazendo artigos, discutindo os trabalhos e brigando também :). Não

posso esquecer das farras que fizemos também, muitas histórias. A Cláudio "Baiano", pelas discussões na melhoria do trabalho e pela sua grande amizade.

A Alessandro Garcia, pelas discas preciosas e ajuda no trabalho.

Aos amigos do laboratório de Engenharia de Software.

Aos meus parceiros de moradia, Diego, Christian, Joelma, Kleinner.

A galera moral de Maceió, Milena, Willy, Marcinho, Bizonho.

Ao departamento de informática, professores e secretaria.

Por fim, ao CNPq pela ajuda financeira. 


\section{Resumo}

Nunes, Camila; Lucena, Carlos (Orientador); Kulesza, Uirá (CoOrientador). Avaliação da Modularidade e Estabilidade de Técnicas de Implementação para Linhas de Produtos de Sistemas Multi-agentes. Rio de Janeiro, 2009. 118p. Dissertação de Mestrado - Departamento de Informática, Pontifícia Universidade Católica do Rio de Janeiro.

Uma Linha de Produtos de Sistemas Multi-agentes (LP-SMA) define uma arquitetura de linha de produto de software (LPS) cujo projeto e implementação são realizados usando agentes de software para compreender features comuns e variáveis. O desenvolvimento de LP-SMA pode ser feito através de plataformas específicas de SMA e técnicas de implementação. Exemplos de tais técnicas são: frameworks orientados a objetos, compilação condicional, arquivos de configuração e programação orientada a aspectos (POA). Todavia, os estudos empíricos existentes não focam na abordagem de LP-SMA, considerando diferentes técnicas de implementação e plataformas específicas de desenvolvimento de SMA. Nesse contexto, este trabalho apresenta uma comparação sistemática de diferentes técnicas de implementação de variabilidades de features de agentes no domínio de LP-SMA. Esta comparação sistemática envolveu o uso de duas plataformas de desenvolvimento de SMA (JADE e Jadex) e das técnicas de implementação: compilação condicional, arquivos de configuração e POA. Neste estudo, um conjunto de métricas de software foram utilizadas para avaliar atributos de qualidade, tais como modularidade e estabilidade. Para a realização deste estudo, duas LP-SMAs foram desenvolvidas. A primeira foi a LP-SMA do Expert Committee, uma linha de produto de sistemas de gerenciamento de conferências. A segunda foi a LP-SMA OLIS, que provê diversos serviços pessoais para os usuários. Os dados coletados durante a realização dos estudos empíricos permitiram o relato de um conjunto de lições aprendidas.

\section{Palavras-chave}

Linhas de Produto de Software. Sistemas Multi-agentes. Estudo Empírico. Técnicas de Implementação. Projeto de Software. 


\section{Abstract}

Nunes, Camila; Lucena, Carlos (Advisor); Kulesza, Uirá (CoAdvisor). Modularity and Stability Assessment of Implementation Techniques for Multi-agent Systems Product Lines. Rio de Janeiro, 2009. 118p. Master Dissertation — Departamento de Informática, Pontifícia Universidade Católica do Rio de Janeiro.

A Multi-agent System Product Line (MAS-PL) defines a Software Product Line (SPL) architecture whose design and implementation are accomplished using software agents to address its common and variable features. The MAS-PL development can be performed through MAS specific platforms and implementation techniques. Examples of such techniques are: object-oriented frameworks, conditional compilation, configuration files and aspect-oriented programming (AOP). However, the existing empirical studies do not focus on MAS-PL approach, considering different implementation techniques and MAS specific platforms. In this context, this work presents a systematic comparison of different variability implementation techniques of agent features in the MAS-PL domain. This systematic comparison involved the use of two platforms of MAS development (JADE and Jadex) and implementation techniques: conditional compilation, configuration files and AOP. In this study, a suite of software metrics were used to evaluate quality attributes, such as modularity and stability. In order to perform this study, two MAS-PLs were developed. The first one was the Expert Committee MAS-PL, a product line of conference management systems. The second one was the OLIS MAS-PL, which provides several personal services to the users. The collected data during the accomplished empirical studies allowed to report a set of lessons learned.

\section{Keywords}

Software Product Lines. Multi-agent Systems. Empirical Study. Implementation Techniques. Software Design. 


\section{Sumário}

1 Introdução $\quad 11$

1.1 Problemática 12

1.2 Limitações dos Trabalhos Relacionados 14

$\begin{array}{lll}1.3 & \text { Solução Proposta } & 15\end{array}$

$\begin{array}{lll}1.4 & \text { Objetivos } & 16\end{array}$

$\begin{array}{lll}1.5 & \text { Estrutura da Dissertação } & 17\end{array}$

2 Linhas de Produtos de Software $r$

2.1 Conceitos 18

2.2 Linhas de Produto de Sistemas Multi-agentes 22

$\begin{array}{lll}2.3 & \text { Resumo } & 23\end{array}$

3 Técnicas de Implementação de Variabilidades $\quad 24$

3.1 Frameworks 24

3.2 Compilação Condicional 25

3.3 Arquivos de Configuração com Injeção de Dependências 26

3.4 Programação Orientada a Aspectos 28

$\begin{array}{ll}3.5 \text { Resumo } & 31\end{array}$

4 Engenharia Empírica e Medição de Software 33

4.1 Conceitos 33

4.2 Métricas 34

4.2.1 Métricas de Separação de Concerns 35

4.2.2 Métricas de Acoplamento 36

4.2.3 Métricas de Coesão 37

4.2.4 Métricas de Tamanho 37

4.2.5 Interação entre Concerns 38

$\begin{array}{lll}4.3 & \text { Resumo } & 38\end{array}$

5 Avaliação da Modularidade e Estabilidade das LP-SMA 40

5.1 Primeiro Experimento 40

5.1.1 Linha de Produto de Sistemas Multi-Agentes do Expert Committee 41

5.1.2 Estrutura do Estudo Experimental 45

5.1.3 Análise da Estabilidade 48

5.1.4 Análise da Modularidade Multi-Releases $\quad 52$

5.2 Segundo Experimento 66

5.2.1 Linha de Produto de Sistemas Multi-Agentes OLIS 66

5.2.2 Estrutura do Estudo Experimental 69

5.2.3 Análise da Estabilidade $\quad 75$

5.2.4 Análise da Modularidade Multi-Releases $\quad 77$

5.2.5 Acoplamento e Coesão 83

5.2.6 Tamanho $\quad 87$

5.2.7 Interação entre Concerns $\quad 87$

$\begin{array}{lll}\text { 5.2.8 } & \text { Interpretação dos dados } & 89\end{array}$ 
5.3 Ameaças à Validação da Análise $\quad 90$

$\begin{array}{lll}5.4 \text { Resumo } & 91\end{array}$

6 Discussões e Lições Aprendidas $\quad 93$

6.1 Benefícios do Estudo 93

6.2 Discussões e Lições Aprendidas 94

6.2.1 Implementação de Papéis 94

6.2.2 Modularidade das features Alternativas $\quad 95$

6.2.3 Estabilidade das Implementações 96

6.2.4 Validação com Testes Estatísticos 96

6.2.5 Evolução das LP-SMA com Orientação a Aspectos 97

6.2.6 Gerenciamento das Features 98

6.2.7 Estratégias de Adoção de Linhas de Produto de Software 98

$\begin{array}{lll}6.3 \text { Resumo } & 99\end{array}$

7 Trabalhos Relacionados $\quad 100$

7.1 Abordagens para Linha de Produtos de Sistemas Multi-agentes 100

7.2 Estudos Empíricos em Linha de Produtos de Software 101

7.3 Estudos Empíricos com Agentes e Aspectos 102

7.4 Diferenças em Relação aos Trabalhos Relacionados 103

8 Conclusões e Trabalhos Futuros 105

$\begin{array}{lll}8.1 \text { Conclusões } & 105\end{array}$

8.2 Contribuições 106

$\begin{array}{ll}\text { 8.3 Trabalhos Futuros } & 108\end{array}$ 


\section{Lista de figuras}

2.1 Notação FODA do modelo de features. 20

2.2 Linhas de Produtos de Software. 20

2.3 Atividades da Linha de Produto. 21

3.1 Implementação de um interesse com POO e POA. 29

4.1 Modelo de Qualidade. 35

5.1 Modelo de Features Simplificado da LP-SMA do EC. 41

5.2 Arquitetura da LP-SMA do EC. 43

5.3 Arquitetura Detalhada da LP-SMA do EC. 43

5.4 Projeto Simplificado OO da LP-SMA do EC. 49

5.5 Projeto Simplificado OA da LP-SMA do EC. $\quad 50$

5.6 Métricas de concern para a feature Revisor. 56

5.7 Métricas de concern para a feature Agentes de Usuário. 58

5.8 Métricas de concern para a feature Agente Notificador. 59

5.9 Métricas de concern para a feature Agente de Prazos/Datas. $\quad 60$

5.10 Métrica CIBC para a feature Revisor. $\quad 61$

5.11 Métrica CIBC para a feature Agentes de Usuário. 62

5.12 Média de Acoplamento do EC. 62

5.13 Média de Coesão do EC. 63

5.14 Métricas de Tamanho da LP-SMA do EC. 65

5.15 Modelo de features do OLIS. $\quad 67$

5.16 Arquitetura da LP-SMA OLIS. 68

5.17 Arquitetura Simplificada da LP-SMA OLIS usando JADE. $\quad 70$

5.18 Arquitetura Simplificada da LP-SMA OLIS OA usando JADE. 71

5.19 Arquitetura Simplificada da LP-SMA OLIS usando Jadex. 72

5.20 Métricas de concern para a feature obrigatória importar eventos. $\quad 79$

5.21 Métricas de concern para a feature alternativa eventos acadêmicos. 81

5.22 Métricas de concern para a feature opcional Sugestão de Eventos. 82

5.23 Métricas de concern para a feature opcional Lembrete de Eventos. 84

5.24 Métricas de concern para a feature opcional Agenda de Eventos. $\quad 85$

5.25 Métrica de acoplamento da LP-SMA OLIS. 86

5.26 Métrica de coesão da LP-SMA OLIS. 86

5.27 Métricas de tamanho da LP-SMA OLIS. 88

5.28 Métrica CIBC para a feature Agentes de Usuário. $\quad 89$ 


\section{Lista de tabelas}

5.1 Funcionalidades do Expert Committee. 42

5.2 Cenários de Mudanças aplicados a LP-SMA do EC. 46

5.3 Propagação das mudanças nas releases do EC. 51

5.4 Releases e Cenários de Mudanças aplicados a LP-SMA OLIS. $\quad 73$

5.5 Propagação das mudanças nas releases do OLIS. 76 